\title{
非常规油浓度分辨菼光光谱多环芳烃的风化特性 研究
}

\author{
王春艳 ${ }^{1,2^{*}},{\text { 黄小东 }{ }^{1}, \text { 㚞心民杨春 }^{2}, \text { 王镇棣 }}^{2}$
}

1. 潍坊学院物理与光电工程学院, 潍坊 261061 ;

2. Emergencies Science and Technology Section (ESTS), Science and Technology Branch, Environment Canada, Ottawa K1A 0H3, Canada

*E-mail: wangcy@bnu.edu.cn

收稿日期：2017-11-13; 接受日期：2018-03-23; 网络版发表日期：2018-04-28 山东省自然科学基金英才计划(编号：ZR2015M007)、山东省自然科学基金培养基金(编号：ZR2018PF016)、国家自然科学基金(批准号： 40706037, 41476081)、山东省博士基金(编号: BS2011HZ015)和山东省重点研发计划(编号: 2016GSF115020)资助

摘要随着常规原油的开采量和运输量的迅速减少, 重质原油、稠油、油砂、油页岩等非常规油逐渐成为海洋 溢油的主体，以往主要针对常规原油检测的技术方法遇到了新的挑战.依据时间分辨荧光光谱的思路，将浓度作 为新的一维坐标引入荧光光谱, 发展了浓度分辨荧光光谱(concentration-resolved-fluorescence spectroscopy, CRFS)，对不同原油样品模拟风化实验、高温馏分实验，结果表明该方法具有很强的抗风化抗干扰能力. 并对相 似稠油原油样品进行了 $120 \mathrm{~d}$ 的光氧化及海水风化实验研究，利用二维Gabor小波分析对视为图像信息的荧光光 谱进行特征提取，结合支持向量机(support vector machine, SVM) 对风化样本进行分类识别，准确率达 $96 \%$, 该方 法快速、准确、经济, 特别适合稠油等非常规溢油的检测, 有望成为溢油排查的重要技术, 也可成为油气地球化 学研究的有效工具之一.

关键词＼cjkstart溢油鉴别, 荧光光谱, 风化, 模拟实验, 多环芳烃

\section{1 引言}

大规模的溢油灾难已经引起了各国政府和社会的 高度重视, 但还应该注意到, 那些不被报道和重视的小 规模漏油溢油事故的频发，其对生态环境破坏的累积 效应，已经与大规模典型溢油事故相当 ${ }^{[1]}$. 这些船舶往 往在肇事后不久就逃离事故现场，以致事故责任的确 认过程中，涉及的溢油嫌疑范围较大，排查耗时费力, 极为困难.
目前常用的分层次溢油鉴别技术主要包含两个层 次: 第一层次是排查技术, 主要包括气相色谱氢火焰检 测器 $\mathrm{GC} / \mathrm{FID}^{[2,3]}$ 、红外光谱 ${ }^{[4,5] \text { 、苂光光谱 }}{ }^{[6,7]}$ 等技术, 于溢油现场取可疑油样和肇事油样，迅速缩小嫌疑样 本数目, 确定重点嫌疑油样; 第二层次详细指纹分析 技术，主要是色谱质谱联用 $(\mathrm{GC} / \mathrm{MS})^{[8,9]}$ 及其衍生技术, 当嫌疑油样指征相似度很大时, 为环境法医学结论提 供重要佐证.

然而，以往主要针对常规原油的检测鉴定的技术

引用格式: 王春艳, 黄小东, 訤心民, 等. 非常规油浓度分辨荧光光谱多环芳烃的风化特性研究. 中国科学: 技术科学, 2018, 48: Wang C Y, Huang X D, Fan X M, et al. Study on weathering characteristic of aromatic hydrocarbons specific to unconditional oil based on concentration-resolved-fluorescence spectroscopy (in Chinese). Sci Sin Tech, 2018, 48: doi: 10.1360/N092017-00391 
方法遇到了新的挑战, 由于开发的石油资源中, 轻质原 油、高品质原油开采和输运量正在迅速减少，重质原 油、稠油、油砂、页岩油等非常规油开采输运量增 加. 但由于常规气相色谱峰容量和分离能力的限制, 当 分析非常规油时，往往会导致色谱基线抬高而形成一 个不可分辨的复杂混合物的大鼓包(unresolved complex mixtures，UCM), 使得低浓度的生物标志物被其 掩盖而无法被检出 ${ }^{[10]}$. 长期以来人们对非常规油成分 中的化合物认识水平远不及正常原油的认识水平 ${ }^{[11,12]}$.

同时，占全球海洋石油污染 $48 \%$ 的事件是与燃料 油有关的，燃料油是原油经过调和以及蒸馏、裂化等 炼制工艺加工而成，引入了人类干预的特征，性质发 生了明显的变化, 很多生物标志化合物往往已经丢失, 涉及船用燃料油泄漏的鉴别及风化研究也鲜有报 道 ${ }^{[13]}$. 现实对海洋溢油样品的识别鉴定、风化评估、 溯源追踪等技术提出了新的指标要求.

特别是排查技术中, GC/FID谱图受风化的影响严 重，操作复杂，红外光谱存在水的强烈吸收和干扰问 题，荧光光谱也存在数据不准确的问题. 而排查的错 误无疑会直接影响最终鉴别结果的准确性，所以着力 于排查技术的发展应当是解决溢油检测问题的重要 思路.

荧光方法作为多环芳烃检测的重要手段，具有灵 敏度高、受风化影响程度小、分析速度快的特点，非 常适用于现场溢油检测操作 ${ }^{[14 \sim 6]}$. 然而常规菼光溢油 鉴别的方法存在准确度不高的问题，这是由于海洋溢 油鉴别过程中海水等其他物质的掺入很难避免，虽然 黄色物质等荧光物质的荧光光谱强度较原油的荧光强 度低很多, 可以忽略不计, 但海水等杂质的存在会对原 油萃取液的浓度造成很大影响，进而对苂光光谱造成 很大影响. 因为一般情况下，原油的苂光光谱随萃取 液浓度的变化比起由油种不同造成的光谱变化还要 大, 因此在浓度不能准确获取的情况下, 无法区别光谱 谱形的变化是由浓度的变化引起的, 还是由油种的不 同引起的 ${ }^{[14 \sim 16]}$.

解决上述问题的一种新思路即：将不容易确定的 浓度固定值扩展成浓度变量. 借鉴时间分辨荧光光 谱 ${ }^{\left[{ }^{17]}\right.}$ 反映苂光光谱在时间上的变化规律的思路，建立 浓度分辨荧光光谱CRFS, 利用由不同芳烃组成的原油 样品, 其荧光光谱随浓度的变化规律不同这一特点, 将 变化规律来作为鉴别指标 ${ }^{[18]}$.
本文对CRFS技术在风化、馏分、海水掺杂等条 件下进行了一系列的模拟实验, 分析重稠油样品的芳 烃组分在上述条件下的鲁棒性和演化特点. 并利用二 维Gabor小波分析结合支持向量机(support vector machine, SVM)对光氧化及海水风化相近溢油样品的 CRFS光谱进行特征提取和分类识别, 探讨其在非常规 油溢油鉴别分析中的应用潜力. 本文的非常规油分析 研究主要涉及重稠油和燃料油, 考虑到非常规原油普 遍具有的轻烃组分少、芳烃含量和碳氢比高等特点, 所以本文提出的非常规油芳烃荧光特性的研究方法应 该具有一定的普适性.

\section{2 实验}

\section{1 浓度分辨谱苂光光谱原理与方法}

目前被广泛使用的荧光光谱技术主要分为二维光 谱和三维光谱，其中二维包括激发光谱、发射光谱和 同步荧光(synchronous fluorescence). 三维荧光包括激 发发射荧光矩阵(excitation-emission-matrix fluorescence, EEMF)和全同步三维光谱(total-synchronousfluorescence, TSF), 实验和理论 ${ }^{[14]}$ 都表明, 同步光谱可 以很好地避免瑞利散射, 二级衍射光谱对样品荧光光 谱信息的掩盖，并不仅仅是激发发射苂光矩阵的坐标 旋转而成, 具有更精确信息.

但无论哪种光谱, 当样品溶液稀释到线性范围后, 这些光谱都仅反映了低环芳烃组分的信息，而低环芳 烃是最容易受风化影响的，特别是对于稠油等轻质烃 类少的非常规原油，仅反映低环芳烃的组分无疑会进 一步降低苂光检测的准确度.

原油中不同环数的多环芳烃(PAHs)其苂光响应的 浓度范围不同，而且当浓度发生变化，各PAHs之间存 在荧光猝灭，因而所构成的荧光光谱随浓度的变化非 常大. 各PAHs之间并不存在一个统一的线性浓度范 围，过高过低的浓度取值只能反映其高环或低环的芳 烃信息, 不能全面地反映样品整体的芳烃构成 ${ }^{[19]}$.

将浓度从一个需要准确的固定值扩展成一维变 量, 不但可以解决现场浓度难以确定的问题, 而且可以 反映不同原油中轻重芳烃组分的苂光随浓度全部响应 特性, 极大地增加了信息量.

由于浓度加上三维光谱构成的四维CRFS光谱无 法展示, 这里选择 $\Delta \lambda=40 \mathrm{~nm}$ 二维同步光谱来表征原油 
的主要光谱信息，所以本文中的讨论都是基于浓度同 步苂光光谱矩阵 (concentration-synchronous-matrix fluorescence, SMF $)^{[20]}$.

如图1所示, 原油样品绥中36-1\#的CSMF苂光光谱 图的不同表现形式. 图1(b)作为Gabor小波分析的图像 处理的对象; 图1(c)可更清晰地反映了不同浓度下同 步光谱的细节信息; 从图1(d)中可见不同的PAHs峰值 随着溶液稀释浓度的降低先升后降变化关系.

\section{2 样品来源与试剂}

油样MCB(Mississippi Canyon Block 807, $\mathrm{API}=17.5$, poured Oct. 21, 2002)蒸发风化模拟实验和 ANS(Alaska North Slope, fresh API=31, poured July 24, 2002)高温馏分实验 ${ }^{[8,9]}$ 的CSMF光谱采集在加拿大环 境部应急科学技术中心溢油实验室完成, 荧光光谱萃 取剂采用Sigma-Aldrich公司的正己烷(色谱纯). 光氧 化及海水风化的相近溢油样品的分类识别实验在中国 海洋大学光学光电子实验室完成, 其中稠油样品“埕北
306\#”(CB)，“旅大A-12\#”(LD)以及“绥中36-1\#”(SZ)由 中国胜利油田录井公司提供. 采用的是天津市光复精 细化工研究所生产的正已烷(色谱纯)作为萃取溶剂.

\section{3 风化实验设计}

溢油事故发生后, 在海洋特有的环境条件下, 有着 复杂的物理、化学和生物变化过程, 并通过这些变化, 最终从海洋环境中消失. 这些变化有扩散、漂移、蒸 发、分散、乳化、光化学氧化分解、沉积以及生物降 解等. 在溢油鉴别中, 检测指标必须具有 “特征差异性” 和“稳定性”, 因而溢油风化模拟实验的设计至关重要.

本研究中模拟实验包含 3 部分: 用于探求指标稳定 性的MCB油样蒸发风化模拟实验、ANS油样高温馏 分实验以及光氧化及海水风化的相近溢油样品的分类 识别实验. 其中, MCB油样模拟实验采用的是旋转蒸 发的实验室油风化技术 ${ }^{[21]}$, 获得 4 个蒸发风化百分比的 $\mathrm{MCB}$ 样本. 通过人为地对油样进行不同程度的风化, 这种技术允许对油蒸发重量丢失进行精确控制. 这一
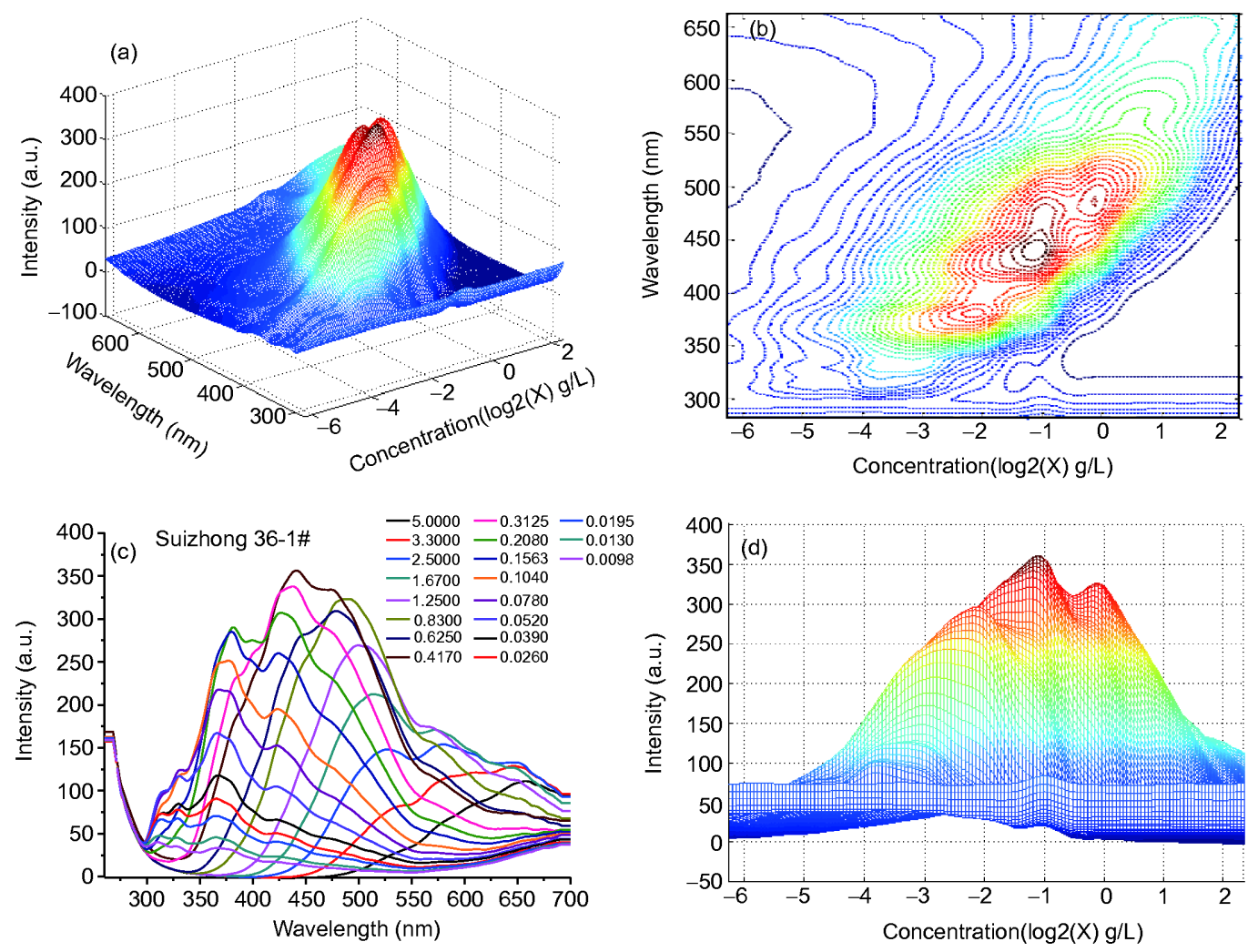

图 1 原油样品绥中36-1\#的CSMF光谱图. (a) CSMF三维图; CSMF光谱强度与浓度二维关系图 
实验设计可实现对检测指标的稳定性的评估 ${ }^{[19]}$.

ANS油样高温馏分实验目的是验证高温馏分的过 程对油样的苂光光谱的影响过程, 采用的是修订了的 ASTM D1160真空馏分方法 ${ }^{[22]}$. 馏分得到的组分为F1: $n$-C6 n-C10范围内的正构烷烃 (初馏点 $\sim 173^{\circ} \mathrm{C}$ ), F2: $n$ C10 n-C16(初馏点174 287 ${ }^{\circ} \mathrm{C}$ ), F3: $n$-C17 n-C34(初馏 点 $\left.288 \sim 481^{\circ} \mathrm{C}\right), \mathrm{F} 4:>n-\mathrm{C} 34\left(\right.$ 初馏点 $\left.>481^{\circ} \mathrm{C}\right)$. 其中 $\mathrm{F} 1$ 对 应轻质组分, F2大致对应于柴油部分, F3大致对应于 燃料油部分, F4对应最重的残渣部分 ${ }^{[19]}$.

为了利用数据分析的方法对非常规溢油苂光鉴别 能力进行评估，进行了光氧化及海水风化的相近溢油 样品的分类识别实验，模拟风化实验分为两个对照组： 海水风化组(烧杯中加入海水, 原油漂浮其上)和未加 海水风化组(直接将原油滴入烧杯中). 考虑到风化周 期较长，将两烧杯置于室内向阳窗台，风化时间均为 $120 \mathrm{~d}$ ，海水取自青岛石老人海水浴场，根据蒸发情况 适量添加, 鉴于原油的苂光光谱的稳定性, 未对样品及 海水做特殊处理. 每个样品形成 36 个风化样本, 3 个油 样共 $3 \times 36=108$ 个样本.

\section{4 仪器和测量方法}

油样MCB蒸发风化模拟实验和ANS高温馏分实 验的荧光光谱的采集使用的是Agilent公司的Cary Eclipse苂光光谱仪, 在 $10^{-4} \sim 40 \mathrm{mg} / \mathrm{mL}$ 的浓度范围内, 按照稀释后浓度/稀释前浓度 $=0.8$ 的稀释倍率将样品 配制成系列浓度的原油萃取液，测量的激发波长和发 射波长的缝宽分别为 5.0 和 $1.5 \mathrm{~nm}$.

光氧化及海水风化溢油样品的分类识别实验中采 用日本岛津公司RF5301苂光分光光度计，测量的激发 波长和发射波长的缝宽分别为 5.0 和 $1.5 \mathrm{~nm}$. 将自然风 化样本分别在 $10^{-4} \sim 10 \mathrm{mg} / \mathrm{mL}$ 的浓度范围内进行稀释, 为简化操作, 按照对半稀释(稀释后浓度/稀释前浓度
$=0.5)$ 的方法获取 18 个系列浓度的原油萃取液. 其中样 品号前面的数字标记为风化时间, 样品号之后加 $\mathrm{h}$ 的表 示引入海水. 实验测量起始激发波长设定为 $220 \mathrm{~nm}$, 起 始发射波长设定为 $260 \mathrm{~nm}$, 即测量最优的波长差 $\Delta \lambda$ $=40 \mathrm{~nm}^{[15]}$ 的同步荧光光谱, 检测荧光光谱范围为 260 700 nm. 分别对各个系列浓度的溢油萃取液获取 其同步光谱, 形成CSMF光谱.

\section{3 结果与讨论}

\section{1 蒸发风化过程对溢油光谱的影响}

溢油泄漏到环境中, 随即发生扩散、挥发、油水 乳化、溶解、光化学氧化、微生物降解 ${ }^{[23,24]}$ 等多种风 化过程, 特别是重度风化过程, 会强烈改变溢油的化学 组成和物理性质，导致油样固有的特征信息受到不同 程度的干扰和破坏, 给溢油鉴别造成了诸多困难 ${ }^{[25,26]}$.

溢油发生后的短时期，蒸发是最重要且占主导的 风化过程 ${ }^{[27]}$. 加拿大环境部对多种原油和石化产品因 不同程度的蒸发风化而引起的化学成分的变化进行了 系统的研究, 主要包括GC-FID色谱研究和GC-MS烷烃 以及芳香烃生物标志物研究, 实验结果可参考相关文 献 $[21,22]$.

对相应的样品进行CSMF荧光光谱分析, 实验结 果表明: 除了高挥发的汽油等轻馏分产品外, 蒸发对 芳烃组分的影响非常小, 即使是对柴油, 其CSMF光谱 也保持了极强的稳定性.

图2所示的是MCB-fresh和MCB-w30.7\%的CSMF 三维光谱, 可见蒸发风化对荧光光谱几乎没有产生影 响. 蒸发损失的质量主要是低分子的烃类，其中低碳 数的正构烷烃蒸发最快, 这部分虽然占蒸发质量的大 部分, 但对荧光光谱没有任何贡献和影响.

芳烃中低环芳烃苯荎系列也会因蒸发而受到损
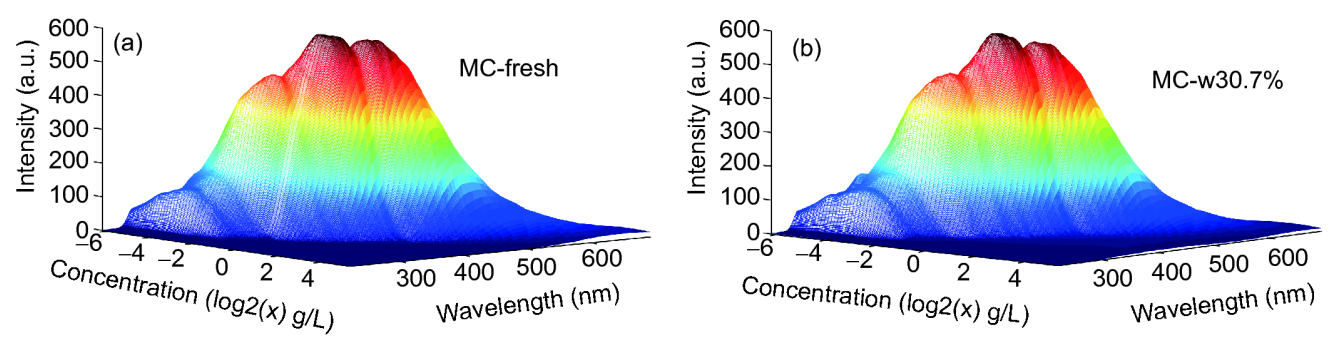

图 2 Mississippi Canyon Block 807的不同风化程度下的CSMF光谱(原油MCB-fresh(a)、风化MCB-w30.7\%(b)) 
失，但由于不同芳烃的苂光浓度响应范围的不同以及 高环芳烃对低环芳烃的荧光猝灭作用，实验表明苯萗 系的小分子芳烃的光谱信息不对CSMF光谱有什么影 响. 前期的汽油和原油 $1: 1$ 混合实验也表明, 汽油的 CSMF光谱对混合后的原油的CSMF光谱没有影响 ${ }^{[28]}$.

这与色谱实验的证据是一致的，蒸发风化产生的 化学组分的变化除了主要包括低分子量正构烷烃的减 少, 还有BTEX, C3-苯的减少甚至完全消失, 但对两个 环的萗及以上的芳烃组分没有影响 ${ }^{[21]}$.

从表1可以看出, 原油样品 $\mathrm{MCB}$ 的fresh样和风化 样之间虽然风化质量损失已经高达 $30.7 \%$, 但其中芳 烃的荧光光谱的差异非常小, 仅 $2.2 \%$, 具有 $97.8 \%$ 的光 谱吻合度. 其中光谱吻合度的计算法采用三维光谱的 归一化后，利用最小二乘法进行谱形差异度计算. 公 式为

$\gamma=\frac{\sum\left(I_{x, y}^{\prime}-I_{x, y}\right)^{2}}{\sum\left(I_{x, y}\right)^{2}}$.

\section{2 馏分过程对溢油光谱的影响}

对ANS 原油不同温度下的高温馏分样品进行 CSMF光谱采集分析，表2为油样馏分的温度信息和对 应光谱所处的主要的波长范围.

图3为原油及不同温度下馏分的石化产物的

表 1 自然风化对原油样品荧光光谱影响

\begin{tabular}{cccc}
\hline 标号Code & $\begin{array}{c}\text { 浓度Conc } \\
(\mathrm{mg} / \mathrm{mL})\end{array}$ & $\begin{array}{c}\text { 风化质量 } \\
\text { 损失 }\end{array}$ & $\begin{array}{c}\text { CSMF光谱 } \\
\text { 差异度 }\end{array}$ \\
\hline MCB-fresh & 80.44 & 0.0 & 0.0 \\
MCB-12.0 & 81.23 & $12.0 \%$ & $1.3 \%$ \\
MCB-19.1 & 79.88 & $19.1 \%$ & $1.6 \%$ \\
MCB-30.7 & 80.73 & $30.7 \%$, & $2.2 \%$ \\
\hline
\end{tabular}

表 2 馏分油样ANS样品及光谱信息

\begin{tabular}{ccc}
\hline 标号Code & 馏分温度 $\left({ }^{\circ} \mathrm{C}\right)$ & 光谱波长范围 $(\mathrm{nm})$ \\
\hline ANS-fresh & - & $260 \sim 553$ \\
ANS-F1 & $\sim 173$ & $230 \sim 300$ \\
ANS-F2 & $173 \sim 287$ & $275 \sim 350$ \\
ANS-F3 & $287 \sim 481$ & $275 \sim 450$ \\
ANS-F4 & $>481$ & $260 \sim 550$ \\
\hline
\end{tabular}

CSMF光谱, 其中图3(a)为ANS原油原样的三维CSMF 图. 为了更好体现光谱主峰波长范围在不同馏分产物 中的变化特性, 图3(b) (f)为三维光谱的强度-波长(intensity-wavelength)的二维展示. 对比分析表明: F1馏 分出来的主要是低环芳烃组分, 与汽油光谱十分相似, 荧光峰在 250 和 $280 \mathrm{~nm}$ 附近, 仅有230 300 $\mathrm{nm}$ 显示出 苯菜系列荧光光谱信息.

F2中包含了更高环数的芳烃组分, 在 275 350 nm 范围内显示 $270,280,300 \mathrm{~nm}$, 反映了二环和三环芳香 烃组分的光谱信息, 以 280 和 $300 \mathrm{~nm}$ 为最高峰值; F3在 275 450 nm波长范围内表现出275, 290, 330, 340, 380, $440 \mathrm{~nm}$ 的CSMF谱峰.

F4为多次馏分后的残留物, 观察其光谱可以发现, 除了 250 300 nm的组分丢失外, 波长300 600 nm范围 内光谱与fresh原油样有着极高的吻合度, 说明了即使 经过 $481^{\circ} \mathrm{C}$ 的高温馏分之后, 高环芳烃的组分比例仍 能保持稳定，这些不易被馏分芳烃组分也即不易被色 谱分离的组分, 对应着最难分离检测的UCM芳烃组 分, 那么从指纹鉴别的角度, 这个波长范围内的CSMF 光谱在馏分环境下具有极强的稳定性, 反映出其极强 的抗风化能力, 可作为非常规溢油鉴别指标可行性. 另一方面, 对其光谱数据的解析角度, 可以避开实验 手段的组分分离, 利用数据挖掘的手段可以解析出芳 烃的组分信息.

此次实验样品采集设计中没有保留各个馏分的剩 余物样品, 无法对各个馏分过程对原油样品的光谱的 影响进行定性定量分析, 下一步的工作将会设计更详 细的馏分对比实验.

\section{3 光氧化及海水风化实验}

对于不同溢油类型的样品, 利用CSMF光谱结合 PCA和PLS等特征提取的方法即可实现 $100 \%$ 的识别 率, 证明了CSMF对于溢油鉴别有很好的特征差异性, 在此不再赘述. 而对于同为渤海湾的三个稠油样本: “埕北306\#”(CB)、“旅大A-12\#”(LD)以及“绥中361\#”(SZ)构成的相似溢油样品集, 再加入光氧化及海水 风化处理后的油源鉴别是极具挑战性的，考虑到该方 法无需脱水等样品的预处理, 其鉴别准确性的意义就 更加明显. 针对海水掺杂风化处理的相似溢油样品, 为了充分利用不同浓度下多环芳烃不同组分的细微差 别作为鉴别依据, 文中借助图像处理的方法, 利用二维 

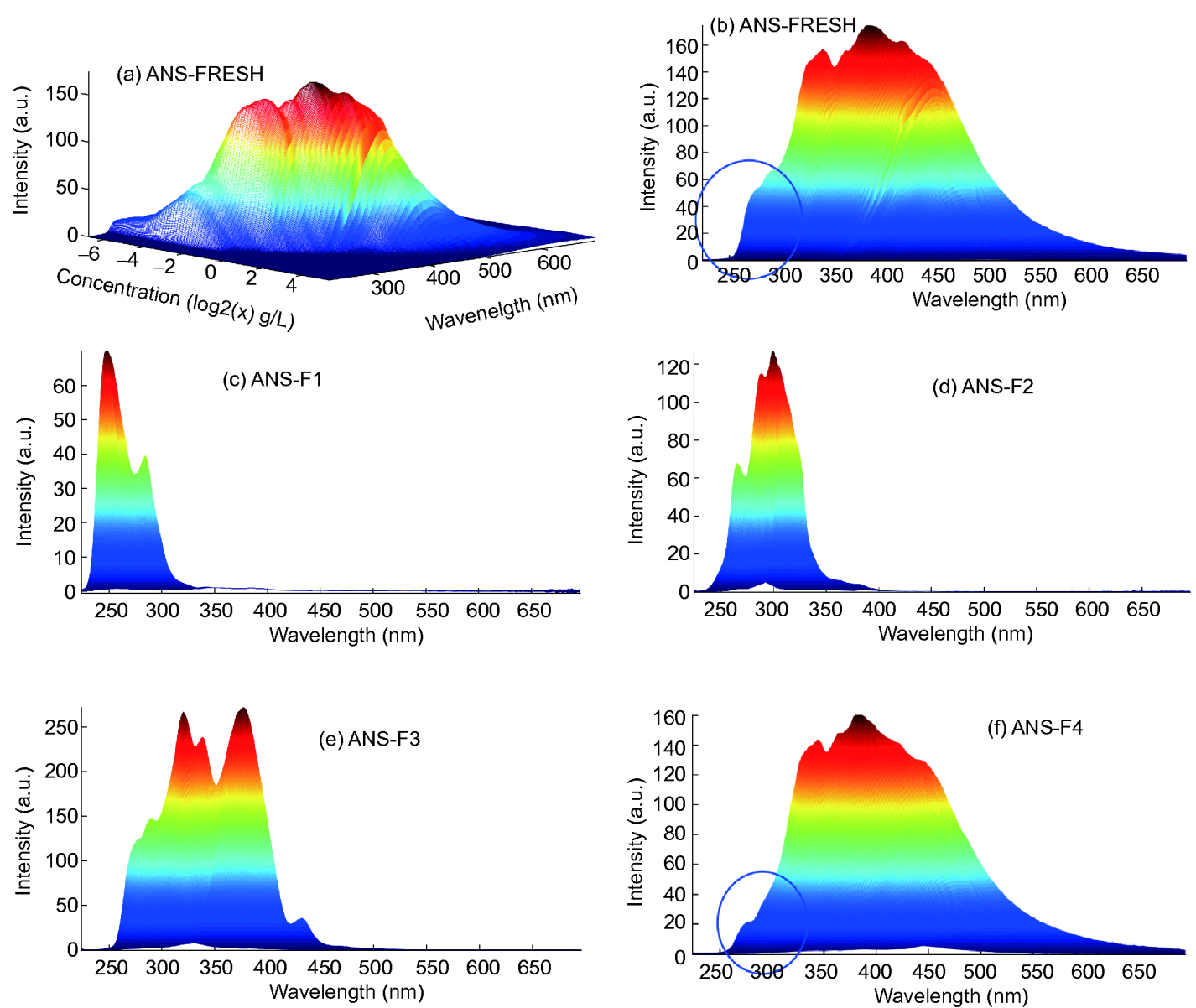

图 3 对ANS原油不同阶段温度馏分石化产物和原油的CSMF光谱对照图

Gabor小波分析对CSMF进行特征提取。这里采用由5 个中心频率和 8 方向的Gabor滤波器组成二维Gabor小 波来提取图像不同的频率尺度和方向的纹理信息.

图4给出的是 3 个油样经过Gabor不同尺度和方向 下的滤波器提取之后得到的特征谱图，其第一行给出 的3个图是未经过Gabor滤波的原始光谱图，第2 5行 分别展示了不同尺寸和方向下Gabor滤波器提取后的 图形特征, 直观的感觉特征图的差异度明显大于原图. 这一方面得益于引入浓度参量后的同步荧光光谱能反 映出更多的不同芳烃组分的荧光信息，另一方面也体 现Gabor对光谱图像表征能力强的优势.

图5给出的是相应的埕北306\#样品的平行样本在 相同的Gabor滤波器下的特征光谱图, 对比图4不同 3 个 油样的Gabor特征谱图可见，利用Gabor分析可以很好 识别不同样品的不同特征，同时平行样本之间有着很 好的稳定性.
二维Gabor滤波器作为带通滤波器在空间域具有 良好的方向选择性，在频率域有良好的频率选择性. Gabor小波变换是通过卷积计算来实现的，卷积是对 图像上给定区域的像素灰度值进行计算以后累加的结 果 ${ }^{[29]}$. 因此, 变换得到的二维Gabor小波变换系数值不 会像像素的灰度值那样随着位置的改变而发生剧烈的 变化. 同时，二维Gabor小波变换能够容许图像特征的 轻微几何变形, 并减小图像噪声的影响. Gabor小波变 换的这些特性十分有利于一定外扰下的 CRFS的鲁棒 表示.

图4和5的对比表明：将CRFS光谱视为图像信息, 利用Gabor小波变换能够捕捉CRFS光谱对应于空间位 置、空间频率及方向选择性的局部结构信息的特性, 实现了对光氧化及海水风化相近原油样品集的有效 的特征提取，为利用 SVM进行分类识别奠定了良好 基础. 

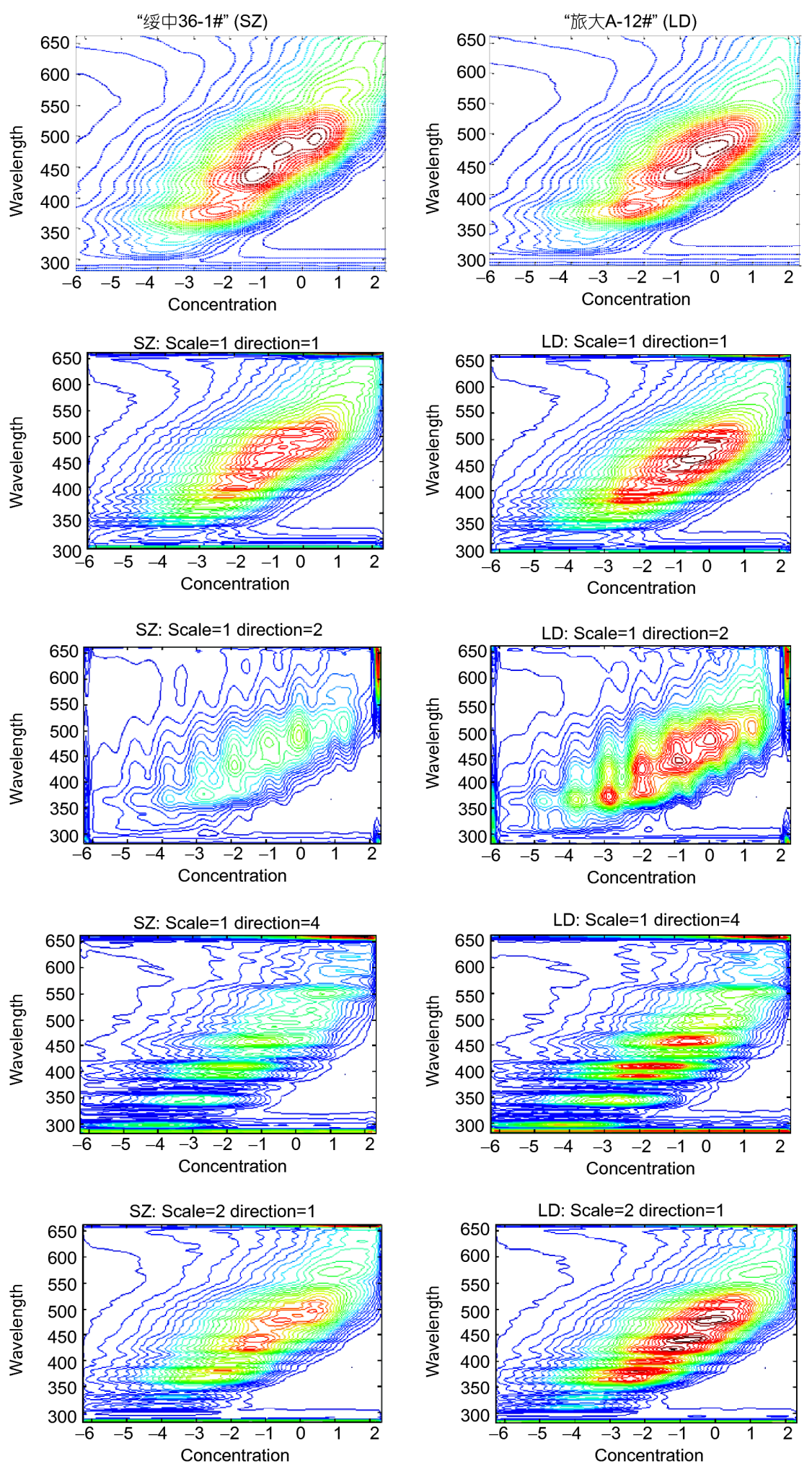
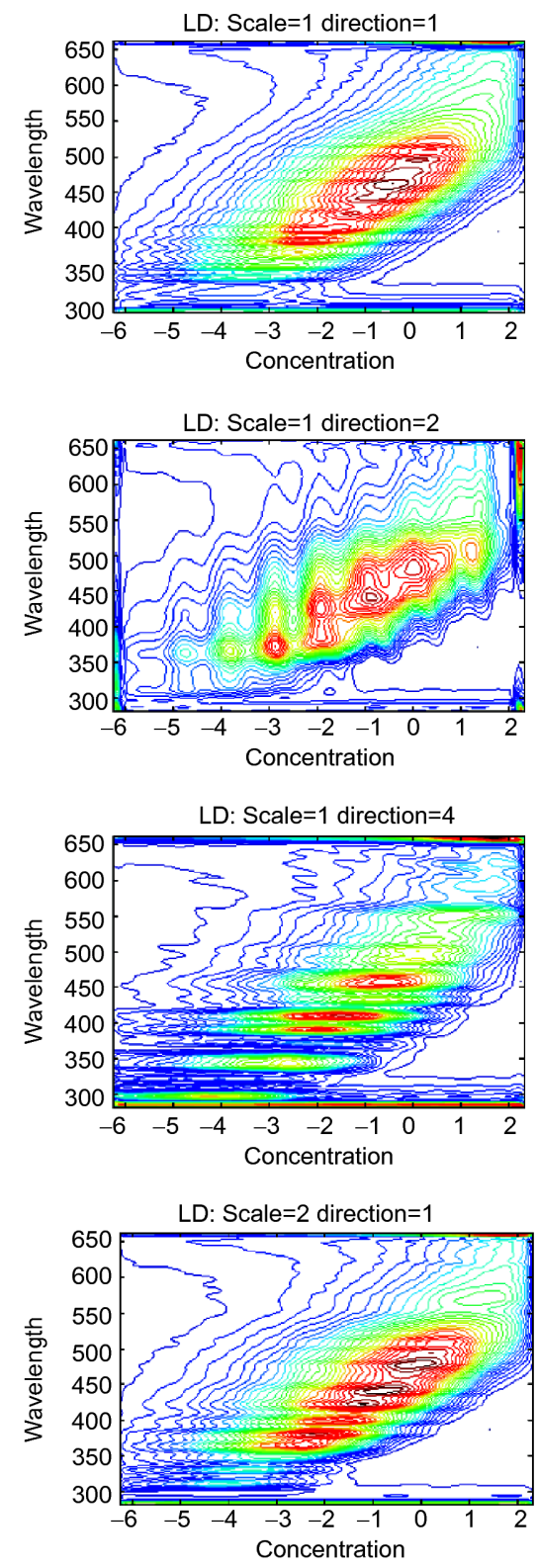

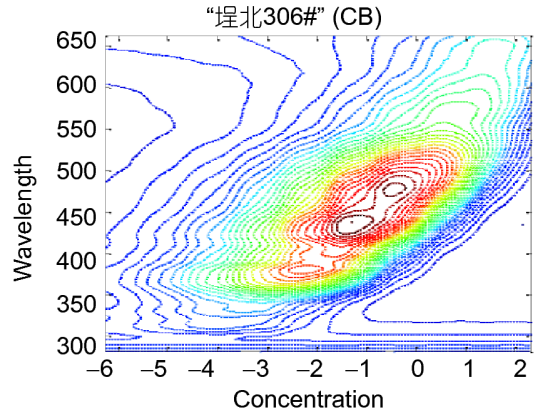

CB: Scale $=1$ direction $=1$
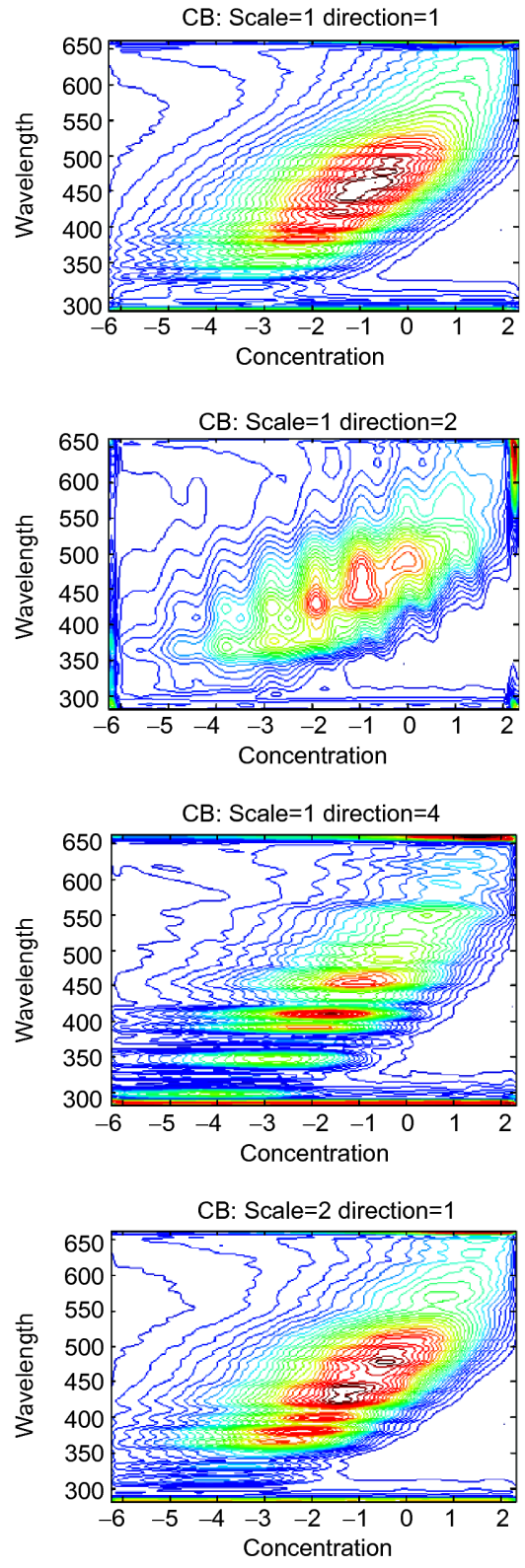

图 4 针对不同的滤波器提取的光氧化及海水风化的相近溢油样品特征光谱比较 

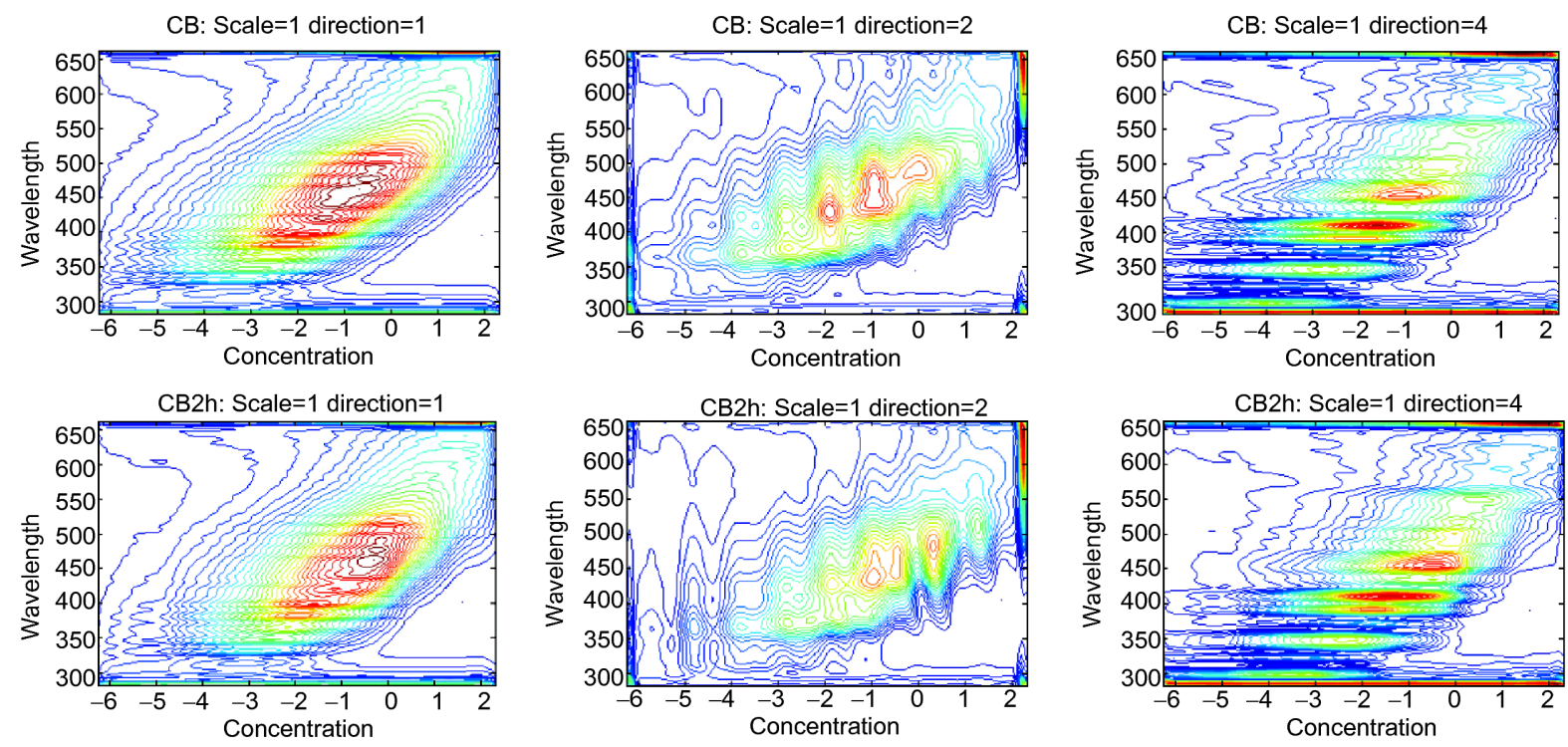

图 5 针对相同的滤波器提取的埕北306\#样品平行样本的特征光谱比较

\subsection{Gabor结合SVM分类识别}

溢油的分类识别采用的SVM是台湾大学林智仁 (Lin Chih-Jen)教授等设计开发的libsvm软件包 ${ }^{[30]}$ ，根 据对测试集分类识别的正确率，应用单因素轮换法， 确定SVM的各主要参数如下表 3 所示. 将Gabor特征提 取后的特征图输入到SVM模式识别的模型中进行分 类识别. 针对光氧化及海水风化的相近溢油样品的 108 个样本进行的留一交叉检验结果表明, 仅有4次判断有 误, 准确率为 $96 \%$. 对照光谱分析识别错误的原因主要 是由对半稀释造成的浓度变化梯度太大，光谱随浓度 变化的信息反应不精细造成的, 如果稀释的配比改为 稀释后浓度/稀释前浓度 $=0.8$, 准确度可以进一步提高.

实验结果与前面的蒸发风化和馏分实验的结果相 似, $120 \mathrm{~d}$ 海水掺杂风化实验结果表明, Gabor小波分析 结合 SVM 可实现小样品集下相近原油CRFS荧光光谱 的信息提取和分类识别的较高的识别的准确率.

\section{4 结论}

本研究提出的浓度分辨荧光光谱(concentrationresolved-fluorescence spectroscopy, CRFS)技术, 针对不 同原油样品进行了蒸发风化实验、高温馏分实验，结 果表明该方法具有很强的抗风化抗干扰能力. 研究还
表 3 SVM的主要参数

\begin{tabular}{cc}
\hline 参数 & 数值 \\
\hline SVM_Type & $0-$ C-SVC \\
Kernel_Type & $1-$ polynomial \\
$-d$ (degree) & 3 \\
$-c$ (cost) & 1 \\
$-p$ (epsilon) & 2 \\
$-g$ (gamma) & 10 \\
$-r$ (coef0) & 1 \\
$-n(\mathrm{nu})$ & 0.5 \\
\hline
\end{tabular}

对 $120 \mathrm{~d}$ 的光氧化及海水风化的相近溢油样品, 并利用 二维Gabor小波分析对视为图像信息的CRFS光谱进行 特征提取, 结合支持向量机(SVM)对风化样本进行分 类识别，准确率达 $96 \%$ ，该方法快速、准确、经济，可 望成为溢油排查的首选技术.

CRFS 光谱不但可以反映更为详细的多环芳烃组 分信息, 而且受风化和海水的影响小, 分析速度快, 灵 敏度高, 十分符合溢油鉴别的要求. 本文为建立快速、 实时、经济、准确的原油指纹鉴别技术提供了很好的 思路. 该方法的发展为非常规溢油检测和中小规模溢 油实时鉴别提供了解决方案, 并有望成为油气地球化 学研究的有效工具之一. 


\section{参考文献}

1 O'Hara P D, Davidson P, Burger A E. Aerial surveillance and oil spill impacts based on beached bird survey data collected in southern British Columbia. Mar Ornithol, 2009, 37: 61-65

2 Wang Z, Yang C, Kelly-Hooper F, et al. Forensic differentiation of biogenic organic compounds from petroleum hydrocarbons in biogenic and petrogenic compounds cross-contaminated soils and sediments. J Chromatogr A, 2009, 1216: 1174-1191

3 Snape I, Harvey P M A, Ferguson S H, et al. Investigation of evaporation and biodegradation of fuel spills in Antarctica I. A chemical approach using GC-FID. Chemosphere, 2005, 61: 1485-1494

4 Fernández-Varela R, Andrade J M, Muniategui S, et al. The comparison of two heavy fuel oils in composition and weathering pattern, based on IR, GC-FID and GC-MS analyses: Application to the Prestige wreackage. Water Res, 2009, 43: 1015-1026

5 Fresco-Rivera P, Fernández-Varela R, Gómez-Carracedo M P, et al. Development of a fast analytical tool to identify oil spillages employing infrared spectral indexes and pattern recognition techniques. Talanta, 2007, 74: 163-175

6 Bugden J B C, Yeung C W, Kepkay P E, et al. Application of ultraviolet fluorometry and excitation-emission matrix spectroscopy (EEMS) to fingerprint oil and chemically dispersed oil in seawater. Mar Pollut Bull, 2008, 56: 677-685

7 Zhou Z, Liu Z, Guo L. Chemical evolution of Macondo crude oil during laboratory degradation as characterized by fluorescence EEMs and hydrocarbon composition. Mar Pollut Bull, 2013, 66: 164-175

8 Yang C, Wang Z, Hollebone B P, et al. Characteristics of bicyclic sesquiterpanes in crude oils and petroleum products. J Chromatogr A, 2009, 1216: 4475-4484

9 Yang C, Wang Z D, Hollebone B P, et al. GC/MS quantitation of diamondoid compounds in crude oils and petroleum products. Environ Forensics, 2006, 7: 377-390

10 王汇夰, 翁娜, 张水昌, 等. 全二维气相色谱/飞行时间质谱与常规色质分析的地球化学参数对比. 中国科学: 地球科学, 2011, 41: 1586-1595

11 王汇殁, 张水昌, 翁娜, 等. 生物标志化合物甾烷、蕉烷的定量分析新方法. 中国科学: 地球科学, 2014, 44: 1713-1722

12 Weng N, Wan S, Wang H, et al. Insight into unresolved complex mixtures of aromatic hydrocarbons in heavy oil via two-dimensional gas chromatography coupled with time-of-flight mass spectrometry analysis. J Chromatogr A, 2015, 1398: 94-107

13 贺世杰, 王传远, 于洪军, 等. 柴油的中重度风化鉴别指标研究. 海洋科学, 2014, 38: 27-32

14 Kumar K, Mishra A K. Analysis of dilute aqueous multifluorophoric mixtures using excitation-emission matrix fluorescence (EEMF) and total synchronous fluorescence (TSF) spectroscopy: A comparative evaluation. Talanta, 2013, 117: 209-220

15 Zhou Z, Guo L, Shiller A M, et al. Characterization of oil components from the Deepwater Horizon oil spill in the Gulf of Mexico using fluorescence EEM and PARAFAC techniques. Mar Chem, 2013, 148: 10-21

16 Divya O, Mishra A K. Multivariate methods on the excitation emission matrix fluorescence spectroscopic data of diesel-kerosene mixtures: A comparative study. Anal Chim Acta, 2007, 592: 82-90

17 Goicoechea H C, Yu S, Moore A F T, et al. Four-way modeling of $4.2 \mathrm{~K}$ time-resolved excitation emission fluorescence data for the quantitation of polycyclic aromatic hydrocarbons in soil samples. Talanta, 2012, 101: 330-336

18 Wang $\mathrm{C}$, Shi X, Li W, et al. Oil species identification technique developed by Gabor wavelet analysis and support vector machine based on concentration-synchronous-matrix-fluorescence spectroscopy. Mar Pollut Bull, 2016, 104: 322-328

19 Wang C, Li W, Luan X, et al. Species identification and concentration quantification of crude oil samples in petroleum exploration using the concentration-synchronous-matrix-fluorescence spectroscopy. Talanta, 2010, 81: 684-691

20 Huang X D, Wang C Y, Fan X M, et al. Oil source recognition technology using concentration-synchronous-matrix-fluorescence spectroscopy combined with 2D wavelet packet and probabilistic neural network. Sci Total Environ, 2018, 616-617: 632-638

21 Wang Z D, Hollebone B, Yang C, et al. Oil composition and properties for oil spill modelling. (US EPA Order No. 3D-6152-NAFX), Ottawa: Environment Canada, 2004

22 Wang Z D. Fractionation and characterization of federated oil. Scotia Light Oil and Their Fractions for the Project of Toxicity Identification and Evaluation of Oil Components Toxic to Laval Fish, ESTD Report 2004-01. Ottawa: Environment Canada, 2004

23 王传远, 侯西勇, 贺世杰, 等. 甾萜烷和多环芳烃在风化溢油鉴别中的应用研究. 海洋环境科学, 2010, 29: 297-302

24 孙培艳, 高振会, 崔文林. 油指纹鉴别技术发展及应用. 北京: 海洋出版社 2007. 116-120

25 Wang C, Gao X, Sun Z, et al. Evaluation of the diagnostic ratios for the identification of spilled oils after biodegradation. Environ Earth Sci, 2013, 
68: 917-926

Wang C, Chen B, Zhang B, et al. Fingerprint and weathering characteristics of crude oils after Dalian oil spill, China. Mar Pollut Bull, 2013, 71: 64-68

27 Bao M, Sun P, Yang X, et al. Biodegradation of marine surface floating crude oil in a large-scale field simulated experiment. Environ Sci-Process Impacts, 2014, 16: 1948-1956

Zhu Z, Jia S, He S, et al. Three-dimensional Gabor feature extraction for hyperspectral imagery classification using a memetic framework. Inf Sci, 2015, 298: 274-287

30 Shen L L, Ji Z. Gabor wavelet selection and SVM classification for object recognition. Acta Automat Sin, 2009, 35: 350-355

\title{
Study on weathering characteristic of aromatic hydrocarbons specific to unconditional oil based on concentration-resolved-fluorescence spectroscopy
}

\author{
WANG ChunYan ${ }^{1,2}$, HUANG XiaoDong ${ }^{1}$, FAN XinMin ${ }^{1}$, YANG Chun ${ }^{2} \&$ WANG ZhenDi ${ }^{2}$ \\ ${ }^{1}$ Department of Physics and Optoelectronic Engineering, Weifang University, Weifang 261061, China; \\ ${ }^{2}$ Emergencies Science and Technology Section (ESTS), Science and Technology Branch, Environment Canada, Ottawa K1A 0H3, Canada
}

With the rapid decline of conventional crude oil's production and transportation, heavy crude oil, heavy oil, oil sands, oil shale and other unconventional oil have gradually become the main body of the marine oil spills. The used oil spill fingerprinting methods mainly for conventional oil are facing new challenges. According to the time-resolved fluorescence spectroscopy, concentration value is introduced as a new data dimension to the fluorescence spectra and the concentration-resolved-fluorescence spectroscopy (CRFS) is developed to express more PAHs information. Laboratory simulation experiments of weathering and distillation were carried out and the results demonstrate this method has strong anti-weathering and anti-interference ability. Oil source classification of closerelated heavy crude oil samples under light weathering and seawater weathering lasting $120 \mathrm{~d}$ are studied. $96 \%$ of the correct rate for the closely related oil source samples is achieved by combining Gabor wavelet with supported vector machine (SVM). The obtained results suggest that the newly-developed method may become specifically applicable in spilled oils identification and also can be applied to other multi-fluorophoric systems such as in petroleum exploration, dissolved organic matter in environment monitoring and petroleum exploration.

oil spill fingerprinting, fluorescence spectroscopy, weathering, simulation experiment, polycyclic aromatic hydrocarbon

doi: $10.1360 /$ N092017-00391 